\title{
Funciones del docente y su práctica reflexiva para la mejora de los aprendizajes en la Universidad
}

\author{
William Jesús Cardenas Zedano \\ william.cardenas@unica.edu.pe \\ https://orcid.org/0000-0003-0296-2935 \\ Universidad Nacional "San Luis Gonzaga" \\ Victor Hugo Herencia Escalante \\ victor.herencia@unica.edu.pe \\ https://orcid.org/0000-0003-1920-4081 \\ Universidad Nacional "San Luis Gonzaga" \\ Sergio Arturo Rojas Chacaltana \\ sergio.rojas@unica.edu.pe \\ https://orcid.org/0000-0001-9312-3533 \\ Universidad Nacional "San Luis Gonzaga" \\ Ica - Perú
}

\section{RESUMEN}

El objetivo del estudio fue determinar la relación entre el rol del docente y su práctica pedagógica reflexiva orientada a mejorar los aprendizajes de los estudiantes de la Facultad de Psicología de una Universidad pública. El estudio es de tipo descriptivo con diseño correlacional, se consideró una muestra de 35 docentes de la Facultad de Psicología de una Universidad Pública en la ciudad de Ica. Se empleó el muestreo de tipo no probabilístico. Para la recolección de los datos, se aplicó la técnica de la encuesta; y como instrumentos se emplearon el Cuestionario sobre el rol del docente universitario y el Cuestionario sobre la praxis reflexiva de Zedano (2019). Los resultados demuestran que existe relación directa entre el rol del docente universitario y la praxis reflexiva; representado en un nivel de significancia de ,000; es decir, que los docentes quienes reflexionan permanentemente acerca de las implicancias de su práctica pedagógica tienden a ejercer procesos de enseñanza mucho más eficaces.

Palabras clave: rol del docente; praxis reflexiva; metodología universitaria. 


\title{
Functions of the teacher and their reflective practice for the improvement of learning at the University
}

\begin{abstract}
El objetivo del estudio fue determinar la relación entre el rol del docente y su práctica pedagógica reflexiva orientada a mejorar los aprendizajes de los estudiantes de la Facultad de Psicología de una Universidad pública. El estudio es de tipo descriptivo con diseño correlacional, se consideró una muestra de 35 docentes de la Facultad de Psicología de una Universidad Pública en la ciudad de Ica. Se empleó el muestreo de tipo no probabilístico. Para la recolección de los datos, se aplicó la técnica de la encuesta; y como instrumentos se emplearon el Cuestionario sobre el rol del docente universitario y el Cuestionario sobre la praxis reflexiva de Zedano (2019). Los resultados demuestran que existe relación directa entre el rol del docente universitario y la praxis reflexiva; representado en un nivel de significancia de ,000; es decir, que los docentes quienes reflexionan permanentemente acerca de las implicancias de su práctica pedagógica tienden a ejercer procesos de enseñanza mucho más eficaces.
\end{abstract}

Key words: Teacher's role; reflective praxis; university methodology.

Artículo recibido: 10 Setiembre. 2021 Aceptado para publicación: 15 octubre. 2021 Correspondencia: william.cardenas@unica.edu.pe Conflictos de Interés: Ninguna que declarar 


\section{INTRODUCCIÓN}

La universidad se constituye en espacio de desarrollo para toda sociedad, en un punto de quiebre para alcanzar el progreso, de formación de los futuros profesionales, para lo cual se requiere que los agentes que laboran en ella cumplan con sus funciones desde una perspectiva de la calidad en el servicio educativo, que los docentes cumplan con sus funciones considerando el desarrollo constante de la ciencia y tecnología, sin perder la perspectiva humanista de su labor. En este contexto universitario se requiere que los docentes cuenten con la actitud de reflexionar sobre su propia labor, lo cual le favorece en la identificación de sus fortalezas y debilidades al momento de desarrollar sus funciones.

Para García (2001), la multidimensionalidad del rol del docente y la complejidad en los diferentes contextos en que se desarrolla en la educación superior, no suele reconocer que, en la mayoría de los instrumentos a emplear en la evaluación, resulta eficiente la experiencia pedagógica. En esta misma línea de estudio, según la perspectiva de Zeichner (2012), "la reflexión representa una valoración para la formación de los saberes que contribuyan al análisis sobre una base regulada de conocimientos en beneficio de una enseñanza de calidad" (p.2).

Respecto al rol del docente universitario, se considera que la docencia universitaria es una de las funciones esenciales de la universidad, sobre la que se apoya la calidad de una universidad y, por ende, la evaluación de la función docente universitaria cobra vital importancia. (Coppola, 2012, p. 83). El rol del docente universitario en el proceso enseñanza aprendizaje es de vital importancia ya que tiene la misión de impartir una formación en valores humanos y sociales y no solo en el conocimiento ya que la formación del estudiante debe ser integral. Puntualizando el compromiso social que deben de tener para que pueden ser respetuosos de la diversidad cultural y del ambiental. Todo esto va determinar la calidad de una universidad.

La función docente debe contribuir a que la educación superior se fortalezca, a través de la función sustantiva de la docencia y la eficacia en el perfil de sus egresados. La función docente abarca la planificación de clase, la implementación de estrategias de enseñanza y la actualización profesional (Vélez y Soto, 2018). Cuando se hace alusión a la función docente, se trata no de una función en el sentido de cumplir unas acciones impuestas a su cargo laboral; aquí el sentido de función va orientado por el efecto que tiene a nivel subjetivo la función del maestro, un maestro con su ser cumple una función reguladora, función 
emancipadora, y una función liberadora. Como parte de las funciones del docente, desde una perspectiva pedagógica, se mencionan: a) provocar el deseo de saber, esto significa que el estudiante se identifique al deseo de saber del docente; que vaya más allá de lo que el docente da en el aula; b) limitar el goce, en tanto la escuela ejerce función reguladora de la pulsión: un maestro que haga pasar del amor al trabajo, que posibilite en el encuentro con el saber una salida adversa a la posición autodestructiva que caracteriza a los jóvenes de hoy; y c) prestar su ser para que el alumno descubra en el mundo de las artes y las ciencias (Vergara, 2015). Para lograrlo, la Universidad debe desarrollar un enfoque basado en competencias para el perfil del docente, ante el contexto actual de globalización y nuevas tecnologías, surge una educación que se divide en competencias que responden a las exigencias de un mundo moderno que brinda una educación mucho más flexible y abierta a los sectores productivos. La aplicación de este perfil, ha originado en muchos países, implementar estrategias con la única finalidad de perfeccionar la calidad educativa como la diversificación de los planes curriculares de estudio y la praxis pedagógica que realiza el docente, a fin de brindar un mejor servicio educativo y de esta manera, obtener profesionales de calidad. (Martell, 2010).

Así mismo, el rol del docente Universitario, se puede dimensionar en:

Investigación: La investigación es constituida como una función esencial y obligatoria de una institución universitaria, que la fomenta y realiza, respondiendo en base a una producción de conocimiento y el desarrollo de tecnologías que responda a las necesidades de la sociedad, con especial énfasis en la realidad del país (Ley 30220, 2017).

Mejoramiento continuo de la enseñanza: El docente debe promover el mejoramiento para que sea continuo para la calidad educativa dentro de la universidad como ente fundamental del desarrollo nacional, de investigación y de cultural (Ley 30220, 2017).

Proyección social: En la educación superior,el tema de proyección social se convierte en un punto de interés social que debe ser articulado desde las instituciones educativas para asumir su responsabilidad.

Gestión universitaria: Hace referencia a la función propia que desarrolla el docente universitario de acuerdo la gestión que realiza. El docente investigador, es un miembro más de la institución, en el cual puede participar activamente en la gestión de su organización o institución. (Torelló, 2011). 
Por otro lado, respecto a la práctica reflexiva, se toma la propuesta de Oyague (2014), quién manifiesta que la universidad peruana no puede ser una institución eficiente educadora y formadora, mientras no tenga un apoyo de las funciones de investigación y extensión. La universidad peruana no alcanza los estándares de calidad debido a que no fortalece la relación entre la investigación y lo referente a la extensión, ya que tener presente esta relación favorece la construcción del conocimiento. Por otro lado, Domingo (2013) señala que la aparición de la práctica reflexiva supone un desafío en cuanto a las habituales metodologías del profesorado universitario. La práctica reflexiva que asumen el docente se da como una actitud sistemática de análisis y valoración de su actuar diaria docente para proponer nuevas estrategias que propicien positivamente en su enseñanza Rodríguez (2013). Para este autor, la práctica que el docente asume en el aula a diaria debe ser autoanalizada y evaluada su accionar hacia sí mismo y así modificar sus estrategias en cada una de sus clases. La praxis reflexiva tiene como propósito fundamental la comprensión y mejora del profesionista que la aplica. En el caso del profesorado, se busca que los docentes logren resignificar su práctica, empoderándolos con nueva información que ellos mismos descubran e investiguen y que les permita estar en una constante prospección de elementos y hallazgos a favor de una mejora personal y profesional continua (Cerecero, 2018).

La función principal de la reflexión es explorar y reflexionar conjuntamente sobre el dilema planteado, así como sobre la relación entre la preocupación identificada y la biografía personal, escolar y educativa de dichas alumnas, es un momento de reflexión y se desarrolla entre el grupo de estudiantes y su profesor. En este espacio reflexivo cada docente ha realizado una pequeña indagación sobre su problema pedagógico (basado en lecturas, observaciones, recabar información del tutor, de expertos, participación en foros, etc.) que es contrastado y discutido con sus colegas. (Saiz y Susinos, 2017). En este sentido Brubacher, Case y Reagan (2000) afirman que volverse un profesional reflexivo es un “compromiso permanente con el crecimiento, el cambio, el desarrollo y el perfeccionamiento.

De ello, surge la necesidad que la universidad desarrolle un Enfoque Reflexivo Docente, según este modelo, la reflexión esta sobre el conocimiento sobre teorías, hechos, etc.; y al conocimiento real practico del día a día (Wallace, 2002). Este enfoque resalta la relevancia de las competencias profesionales como punto de inicio en el desarrollo de habilidades y capacidades educativas. También cobra relevancia los saberes previos de los estudiantes, ya 
que servirán como base en la construcción de nuevos conocimientos. La práctica reflexiva resalta el saber del docente desde su quehacer educativo en las aulas, sobre una reflexión sobre sí mismo. (Schön, 1998).

Desde la perspectiva de Sparks-Langer y Colton (en Brubacher, Case y Reagan, 2000), afirma que la practica reflexiva, se dimensiona en:

El elemento cognitivo, que incluye aquellos conocimientos que sustentan las distintas decisiones que toma el docente. El pensamiento reflexivo sobre lo que enseña en sus actividades académicas, el cómo imparte los nuevos conocimientos a sus estudiantes y tomar mejores decisiones en el aula si así lo requiera para la solución de problemas. Según Shulman (1987) dichas instrucciones aluden al fundamento cognitivo del docente en su trabajo diario y son necesarios para una práctica de la enseñanza reflexiva.

El elemento crítico, que comprende diversos aspectos referidos a la moralidad y la ética de la comprensión. Este elemento de pensamiento reflexivo se basa en la relación a los aspectos morales y éticos de la compasión y la justicia social (Sparks-Langer y Colton 1991).

El elemento narrativo, se refiere a la reconstrucción de la realidad de acuerdo a los relatos de la propia experiencia en la que se contextualizan la reflexión. Este elemento alude al pensamiento reflexivo. Los relatos que el docente realiza sobre sus experiencias diarias presentan muchas formas y a la vez cumplen diversas funciones. Aquí los docentes mencionan que la redacción diaria de sus experiencias ayuda a construir su pensamiento reflexivo siendo su actuar más reflexivo.

En la actualidad, algunos docentes universitarios muestran ciertas debilidades en relación al cumplimiento de su rol docente, las cuales se presentan en el campo de la investigación, mostrando limitaciones en sus capacidades investigativas. Muchos docentes se capacitan por su cuenta, pero es necesario apoyo por parte de la universidad brindar programas de especialización en algunas asignaturas e incentivar a sus docentes por conseguir una profesionalización de calidad. Otro de los aspectos identificados está referido a la predisposición de mejoramiento continuo de la enseñanza y el trabajo de proyección social que debe promover en su contexto social y la gestión universitaria que son los procedimientos en los cuales se involucra en bien de la institución lo cual no se observa. Por otro lado, está la práctica reflexiva en la cual se puede identificar dificultades en el elemento cognitivo, crítico y narrativo. Por lo tanto, consideramos que aquellos docentes quienes 
presentan un adecuado rol docente respecto a las funciones que le son inherentes, tienen una mejor práctica reflexiva sobre el rol que desempeñan.

El objetivo del estudio fue determinar la relación existente entre las funciones del docente universitario y su práctica reflexiva en docentes de la Facultad de Psicología de una Universidad Pública en la ciudad de Ica, mediante un análisis de correlación bivariada, con la finalidad de conocer el comportamiento de las variables de estudio.

\section{MATERIALES Y MÉTODOS}

\section{Diseño}

El estudio es de tipo no-experimental con diseño correlacional. Desde la propuesta de Hernández, Fernández \& Baptista (2014) manifiestan que "los estudios correlacionales, cumplen el propósito de establecer el posible vínculo o la relación entre componentes, categorías o variables de estudio de un fenómeno". Asimismo, es de carácter cuantitativo, de corte transversal; se enfocó en la búsqueda y recolección de datos numéricos, los cuales fueron procesados estadísticamente, según las variables.

\section{Participantes}

En el desarrollo del estudio participaron 35 docentes adscritos a la Facultad de Psicología de una Universidad Pública de Ica, cuyas edades estaban comprendidas entre los 28 a 60 años. La selección de la muestra se realizó a través de la aplicación de la técnica del muestreo de tipo no probabilístico.

\section{Instrumentos}

\section{Cuestionario sobre las funciones del docente universitario}

Para la recolección de los datos se diseñó el instrumento Cuestionario sobre las funciones del docente universitario elaborado por Zedano (2019). Respecto a las funciones del docente universitario fueron evaluadas mediante las dimensiones investigación, mejoramiento continuo de la enseñanza, proyección social, gestión universitaria. El instrumento consta de 24 ítems con opciones de respuesta de siempre a veces nunca, con un puntaje máximo de 72 puntos. Los resultados de la variable se categorizaron en Deficiente [24-36>/Regular [3648> / Bueno [48-60> / Muy bueno [60-72].

\section{Cuestionario sobre la práctica reflexiva}

Se diseñó el instrumento Cuestionario sobre la práctica reflexiva elaborado por Zedano (2019), dividido en sus dimensiones por elemento cognitivo, elemento crítico, elemento narrativo, constituido por 24 ítems, con opciones de respuesta de siempre a veces nunca, con 
puntaje máximo de 72 puntos. Los resultados de la variable se categorizaron en Bajo [2436> / Medio [36-48> / Alto [48-60> / Muy alto [60-72]

Ambos instrumentos fueron sometidos a la prueba de fiabilidad interna de Alpha de Cronbrach con un valor mayor igual a $\alpha$ : 0.8 . considera que el instrumento es confiable.

\section{Tabla 1}

Cuestionario sobre las funciones del docente universitario

Estadísticas de fiabilidad

\begin{tabular}{|r|r|r|}
\hline & $\begin{array}{c}\text { Alfa de } \\
\text { Cronbach } \\
\text { basada en } \\
\text { elementos } \\
\text { Alfa de } \\
\text { Cronbach }\end{array}$ & \\
\hline, 907 &, 915 & \\
estandarizados & N de elementos \\
\hline
\end{tabular}

\section{Tabla 2}

Cuestionario sobre la práctica reflexiva

Estadísticas de fiabilidad

\begin{tabular}{|c|c|c|}
\hline $\begin{array}{c}\text { Alfa de } \\
\text { Cronbach }\end{array}$ & $\begin{array}{c}\text { Alfa de } \\
\text { Cronbach } \\
\text { basada en } \\
\text { elementos } \\
\text { estandarizados }\end{array}$ & $\mathrm{N}$ de elementos \\
\hline ,896 &, 902 & 24 \\
\hline
\end{tabular}

La validez de contenido se logró mediante la consulta de experto (técnica juicio de expertos) quien determina si el instrumento en este caso el cuestionario reúne las condiciones necesarias para ser aplicado.

El cuestionario sobre las funciones del docente universitario paso por juicio de expertos quienes consideraron el instrumento como bueno.

El cuestionario sobre la práctica reflexiva paso por juicio de experto quienes consideraron el instrumento como bueno. 


\section{RESULTADOS Y DISCUSIÓN}

\section{Figura 1}

Nivel de desarrollo de las funciones del docente universitario en la facultad de psicología de la universidad nacional "San Luis Gonzaga" - Ica.

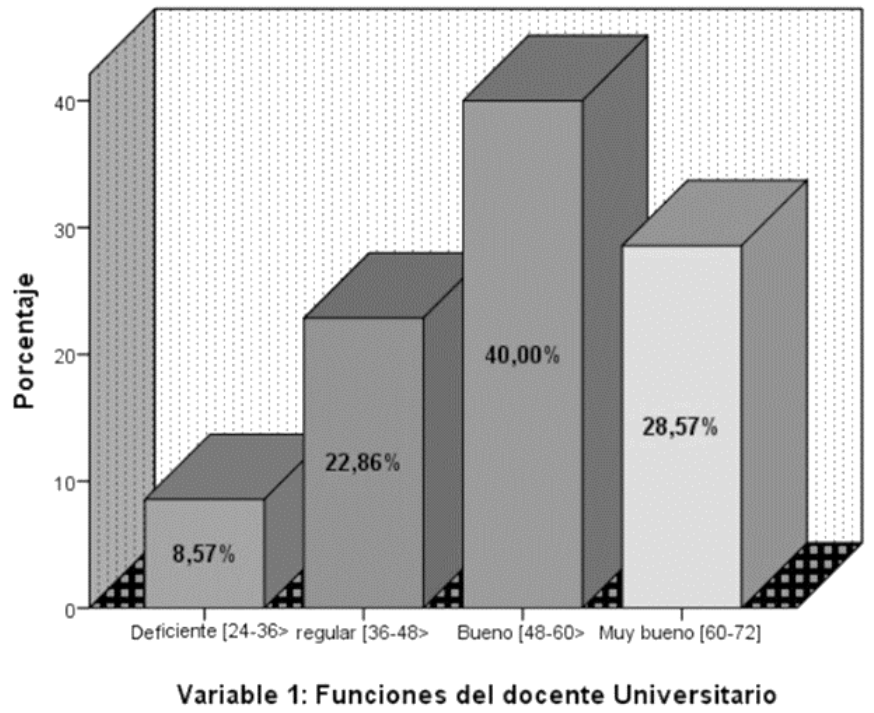

\section{Tabla 3}

Nivel de desarrollo de las funciones del docente universitario en la facultad de psicología de la universidad nacional "San Luis Gonzaga" - Ica

\begin{tabular}{crrrrr}
\hline \hline Categorias de la variable & Frecuencia & Porcentaje & $\begin{array}{c}\text { Porcentaje } \\
\text { válido }\end{array}$ & $\begin{array}{r}\text { Porcentaje } \\
\text { acumulado }\end{array}$ \\
\hline Válido & Deficiente [24-36> & 3 & 8,6 & 8,6 & 8,6 \\
& regular [36-48> & 8 & 22,9 & 22,9 & 31,4 \\
& Bueno [48-60> & 14 & 40,0 & 40,0 & 71,4 \\
Muy bueno [60-72] & 10 & 28,6 & 28,6 & 100,0 \\
Total & 35 & 100,0 & 100,0 & \\
\hline \hline
\end{tabular}

Fuente: Base de datos cuestionario de las funciones del docente universitario

En la tabla se puede observar que el 8,57\% (3) de los docentes presentan un nivel deficiente de desarrollo de las funciones del docente universitario; un 22,9\% (8) de los docentes presentan un nivel regular de desarrollo de las funciones del docente universitario; un 40,0\% (14) de los docentes presentan un nivel bueno de desarrollo de las funciones del docente universitario; y un 28,6\% (10) de los docentes presentan un nivel muy bueno de desarrollo de las funciones del docente universitario. 
Como se puede apreciar la mayoría se ubica en la categoría bueno lo que demuestra que de manera general que los docentes presentan un nivel muy bueno de desarrollo de las funciones del docente universitario.

\section{Figura 2}

Nivel de práctica reflexiva en docentes de la facultad de psicología de la universidad nacional "San Luis Gonzaga" - Ica

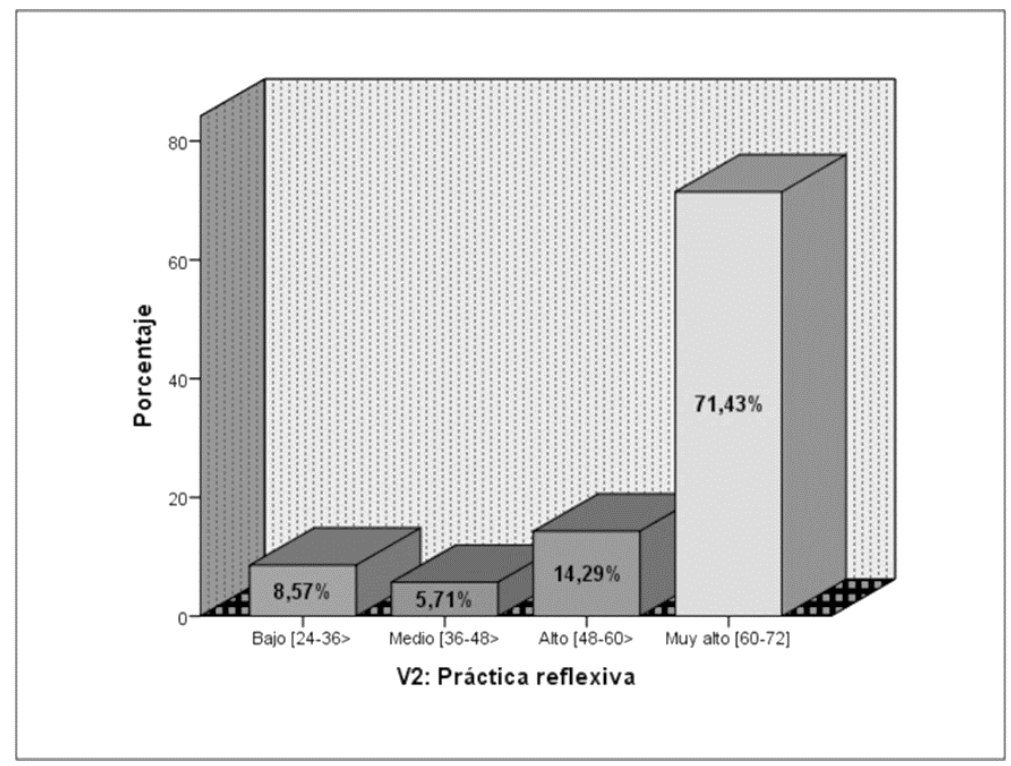

\section{Tabla 4}

Nivel de práctica reflexiva en docentes de la facultad de psicología de la universidad nacional "San Luis Gonzaga" - Ica

\begin{tabular}{lrrrrr}
\hline \hline Categorías de la variable & Frecuencia & Porcentaje & $\begin{array}{c}\text { Porcentaje } \\
\text { válido }\end{array}$ & $\begin{array}{r}\text { Porcentaje } \\
\text { acumulado }\end{array}$ \\
\hline Válido & Bajo [24-36> & 3 & 8,6 & 8,6 & 8,6 \\
& Medio [36-48> & 2 & 5,7 & 5,7 & 14,3 \\
& Alto [48-60> & 5 & 14,3 & 14,3 & 28,6 \\
& Muy alto [60-72] & 25 & 71,4 & 71,4 & 100,0 \\
& Total & 35 & 100,0 & 100,0 & \\
\hline \hline
\end{tabular}

Fuente: Base de datos cuestionario sobre práctica reflexiva

En la tabla, se puede observar que el 8,6\% (3) de los docentes presentan un nivel bajo de práctica reflexiva; un $5,7 \%$ (2) de los docentes presentan un nivel medio de práctica 
reflexiva; un 14,3\% (5) de los docentes presentan un nivel alto de práctica reflexiva; y un $71,4 \%$ (25) de los docentes presentan un nivel muy alto de la práctica reflexiva.

Como se puede apreciar la mayoría se ubica en la categoría muy alto lo que demuestra de manera general que los docentes presentan un nivel muy alto con respecto a la práctica reflexiva.

\section{Tabla 5}

Correlación de Rho de Spearman entre las variables de estudio funciones del docente universitario y práctica reflexiva endocentes de la facultad de psicología de la universidad nacional "San Luis Gonzaga" - Ica

\begin{tabular}{|c|c|c|c|c|c|c|}
\hline \multicolumn{7}{|c|}{ Correlaciones } \\
\hline & & & $\begin{array}{l}\text { D1: Elemento } \\
\text { cognitivo }\end{array}$ & $\begin{array}{l}\text { D2: Elemento } \\
\text { crítico }\end{array}$ & $\begin{array}{l}\text { D3: Elemento } \\
\text { narrativo }\end{array}$ & $\begin{array}{l}\text { VY: Práctica } \\
\text { reflexiva }\end{array}$ \\
\hline \multirow[t]{15}{*}{ Rho de Spearman } & D1: Investigación & $\begin{array}{l}\text { Coeficiente de } \\
\text { correlación }\end{array}$ &, $385^{\circ}$ &, 214 &, 274 &, 275 \\
\hline & & Sig. (bilateral) & 022 & 217 &, 112 & ,109 \\
\hline & & $\mathrm{N}$ & 35 & 35 & 35 & 35 \\
\hline & $\begin{array}{l}\text { D2: Mejoramiento } \\
\text { continuo de la enseñanza }\end{array}$ & $\begin{array}{l}\text { Coeficiente de } \\
\text { correlación }\end{array}$ &, $523^{*}$ &, $562^{*}$ &, $684^{*}$ &, $648^{-\infty}$ \\
\hline & & Sig. (bilateral) & ,001 &, 000 &, 000 & ,000 \\
\hline & & $\mathrm{N}$ & 35 & 35 & 35 & 35 \\
\hline & D3: Proyección social & $\begin{array}{l}\text { Coeficiente de } \\
\text { correlación }\end{array}$ &, $376^{\circ}$ & $473^{*}$ &, $477^{*}$ & $467^{\circ}$ \\
\hline & & Sig. (bilateral) &, 026 &, 004 &, 004 &, 005 \\
\hline & & $\mathrm{N}$ & 35 & 35 & 35 & 35 \\
\hline & D4: Gestión Universitaria & $\begin{array}{l}\text { Coeficiente de } \\
\text { correlación }\end{array}$ & $689^{\circ}$ &, $597^{\circ}$ &, $592^{*}$ & $645^{\circ}$ \\
\hline & & Sig. (bilateral) &, 000 &, 000 &, 000 &, 000 \\
\hline & & $\mathrm{N}$ & 35 & 35 & 35 & 35 \\
\hline & $\begin{array}{l}\text { VX: Funciones del } \\
\text { docente Universitario }\end{array}$ & $\begin{array}{l}\text { Coeficiente de } \\
\text { correlación }\end{array}$ & ,622" &, $588^{*}$ &, $655^{*}$ & $653^{-}$ \\
\hline & & Sig. (bilateral) &, 000 &, 000 &, 000 &, 000 \\
\hline & & $\mathrm{N}$ & 35 & 35 & 35 & 35 \\
\hline
\end{tabular}

Fuente: Data de resultados con la aplicación del cuestionario sobre las funciones del docente universitario y el cuestionario sobre la práctica reflexiva

\section{En la tabla 5, se deduce lo siguiente:}

Existe relación significativa entre las funciones del docente universitario y la práctica reflexiva según el nivel de significancia de ,000; así mismo existe una relación significativa entre las dimensiones de la variable funciones del docente universitario y la variable práctica reflexiva se detalla a continuación:

Existe una relación significativa entre funciones del docente universitario y el elemento cognitivo según el nivel de significancia de ,000.

Existe una relación significativa entre funciones del docente universitario y el elemento crítico según el nivel de significancia de, 000 .

Existe una relación significativa entre funciones del docente universitario y el elemento narrativo según el nivel de significancia de, 000 . 
Existe una relación poco significativa entre la investigación y la práctica reflexiva según el nivel de significancia de, 109 .

Existe una relación significativa entre el mejoramiento continuo de la enseñanza y la práctica reflexiva según el nivel de significancia de, 000 .

Existe una relación significativa entre la proyección social y la práctica reflexiva según el nivel de significancia de ,005 Existe una relación significativa entre la gestión universitaria y la práctica reflexiva según el nivel de significancia de, 000 .

\section{Tabla 6}

Coeficiente de correlación Rho Spearman entre las funciones del docente universitario y la práctica reflexiva

\begin{tabular}{|c|c|c|c|c|}
\hline \multicolumn{5}{|c|}{ Correlaciones } \\
\hline & & & $\begin{array}{l}\text { V1: Funciones } \\
\text { del docente } \\
\text { Universitario }\end{array}$ & $\begin{array}{l}\text { V2: Práctica } \\
\text { reflexiva }\end{array}$ \\
\hline \multirow{6}{*}{$\begin{array}{l}\text { Rho de } \\
\text { Spearman }\end{array}$} & \multirow{3}{*}{$\begin{array}{l}\text { V1: Funciones del docente } \\
\text { Universitario }\end{array}$} & Coeficiente de correlación & 1,000 & $653^{\circ}$ \\
\hline & & Sig. (bilateral) & &, 000 \\
\hline & & $\mathrm{N}$ & 35 & 35 \\
\hline & \multirow[t]{3}{*}{ V2: Práctica reflexiva } & Coeficiente de correlación &, $653^{*}$ & 1,000 \\
\hline & & Sig. (bilateral) &, 000 & \\
\hline & & $\mathrm{N}$ & 35 & 35 \\
\hline
\end{tabular}

Los datos recogidos con los instrumentos, se trasladó al programa estadístico SPSS versión 24 y obteniendo como resultado que sí existe relación directa entre las funciones del docente universitario y la práctica reflexiva en la facultad de psicología de la Universidad Nacional "San Luis Gonzaga" de Ica; esta relación representa un 0,653. El hecho que resulta un valor positivo (el coeficiente de correlación simple) se comprueba que a un adecuado nivel de funciones del docente universitario le corresponde un adecuado nivel de práctica reflexiva; a un inadecuado nivel de funciones del docente universitario le corresponde un inadecuado nivel de práctica reflexiva.

\section{DISCUSIÓN}

Los resultados demuestran que existe relación directa entre las funciones del docente universitario y el elemento cognitivo de la práctica reflexiva. Esto se valida al obtener un 
nivel de significancia de 0,000 (Tabla 3) que refleja una correlación significativa es decir a un adecuado funciones del docente universitario le corresponde un adecuado nivel del elemento cognitivo; a un inadecuado nivel de funciones del docente universitario, le corresponde un inadecuado nivel del elemento cognitivo; estos resultados se evidencia de una u otra manera a lo encontrado por Santivañez (2014), en donde señala que las estrategias que asume el docente correspondiente a su espacio que es el aula donde se desarrolla; con apoyo del currículo permite comprender y dar solución a diferentes problemáticas. Respecto a la información sustentada en el marco teórico se tiene a Sparks-Langer y Colton (en Brubacher, Case \& Reagan, 2000), quienes definen el elemento cognitivo como el pensamiento reflexivo sobre lo que enseña en sus actividades académicas. Respecto a la relación directa existente entre las funciones del docente universitario y el elemento crítico de la práctica reflexiva, valida al obtener un nivel de significancia de 0,000 (Tabla 3) que refleja una correlación significativa es decir a un adecuado rol de las funciones del docente universitario le corresponde un adecuado nivel del elemento crítico; a un inadecuado nivel de funciones del docente universitario, le corresponde un inadecuado nivel del elemento crítico. Estos resultados pueden ser contrastados con la investigación realizada por Reynaldo, La Torre y Frisancho (2011), en donde señala que al asumir una reflexión basada en sus habilidades como docente esto daría un largo camino que recorrer para mejorar sus actividades en clase y poder a través de esta reflexión mejorar su actuar. En el marco teórico se tiene a Sparks-Langer y Colton (en Brubacher, Case y Reagan, 2000), quienes definen el elemento crítico como diversos aspectos referidos a la moralidad y la ética de la comprensión. Por otro lado, se ha logrado determinar que existe relación directa entre las funciones del docente universitario y el elemento narrativo de la práctica reflexiva, esto se observa al obtener un nivel de significancia de ,000. (Tabla 3) que refleja una correlación significativa es decir a un adecuado nivel de funciones del docente universitario le corresponde un adecuado nivel del elemento narrativo; a un inadecuado nivel de funciones del docente universitario, le corresponde un inadecuado nivel del elemento narrativo. Lo referido puede ser contrastados con la investigación realizada por Rodríguez (2013), quien señala que los niveles de reflexión dieron un análisis de que los docentes dieran pautas para la formulación de indicadores sobre la práctica reflexiva, pues a través de sus diferentes experiencias podrán seguir reforzando su proceso de enseñanza. Respecto a la información sustentada en el marco teórico se tiene a Sparks-Langer y Colton (en Brubacher, Case y 
Reagan, 2000), quienes definen el elemento narrativo como una reconstrucción de la realidad a través de relatos de la experiencia propia que contextualizan la reflexión. Se ha logrado determinar que existe relación directa entre la investigación y la práctica reflexiva. Esta hipótesis se valida al obtener un nivel de significancia de ,109 (Tabla 3) que refleja una correlación es decir a un adecuado nivel de investigación le correspondería un adecuado nivel de práctica reflexiva; a un inadecuado nivel de investigación le corresponde un inadecuado nivel de práctica reflexiva. En estos resultados si bien existe una relación es a la vez una relación poco significativa; esta reflexión ase alusión al trabajo de Tolentino (2014). Quien hace referencia al desempeño académico depende del desarrollo profesional y la investigación que realice sobre su especialidad para mejorar su trabajo. Respecto a la información sustentada en el marco teórico esta fue fundamentada por la Ley N 30220 ley universitaria del capítulo VIII, quien define la investigación como algo esencial y de forma obligatoria en la universidad, en la cual se fomenta y realiza, respondiendo a la producción de conocimiento y desarrollo de tecnologías de acuerdo a las necesidades de la sociedad, con énfasis en la realidad nacional.

También se logró demostrar que existe relación directa entre el mejoramiento continuo de la enseñanza y la práctica reflexiva en la facultad de psicología de la Universidad Nacional "San Luis Gonzaga" de Ica. Esta hipótesis se valida al obtener un nivel de significancia de ,000 (Tabla 3) que refleja una correlación significativa es decir a un adecuado nivel de mejoramiento continuo de la enseñanza le corresponde un adecuado nivel de práctica reflexiva; a un inadecuado nivel de mejoramiento continuo de la enseñanza le corresponde un inadecuado nivel de práctica reflexiva. Se contrasta con la investigación realizada por Coppola (2012), en donde concluyo que cada país donde se hizo el estudio se concluye que se debería asumir una nueva gestión estratégica con cambios en la educación superior con carácter innovador en lo institucional y pedagógico, esto conlleva a que los docentes deben estar en constante capacitación y actualización. El sustento teórico se contrasta con lo señalado en la Ley No 30220 ley universitaria del Capítulo VIII, en donde se considera que el mejoramiento continuo de la enseñanza es en donde el docente debe promover el mejoramiento continuo para la calidad educativa dentro de la universidad como ente fundamental del desarrollo a nivel nacional, de investigación y de cultural.

Así mismo, se ha logrado determinar que existe relación directa entre la proyección social y la práctica reflexiva en la facultad de psicología de la Universidad Nacional "San Luis 
Gonzaga" de Ica. Esta hipótesis se valida al obtener un nivel de significancia de ,005 (Tabla 3) que refleja una correlación es decir a un adecuado nivel de proyección social le corresponde un adecuado nivel de práctica reflexiva; a un inadecuado nivel de proyección social le corresponde un inadecuado nivel de práctica reflexiva. Esto guarda relación con la propuesta de Reynaldo, La Torre y Frisancho (2011) quien menciona la importancia de la reflexión sobre su trabajo y hacía donde más va, cuál es su extensión, esa extensión es menciona en el presente estudio sobre el desarrollo de la práctica de la proyección social. Respecto a la información sustentada en el marco teórico esta fue fundamentada por la LEY No 30220 ley universitaria del capítulo VIII, en donde la proyección social se convierte en un punto de interés social que debe ser articulado desde las instituciones educativas para asumir su responsabilidad. El trabajo realizado por los docentes cuenta con buen apoyo, pero aun es necesario seguir el trabajo para obtener mejores resultados.

Finalmente; se ha logrado determinar que existe relación directa entre la gestión universitaria y la práctica reflexiva en la facultad de psicología de la Universidad Nacional "San Luis Gonzaga" de Ica. Esta hipótesis se valida al obtener un nivel de significancia de ,000 (Tabla 3) que refleja una correlación es decir a un adecuado nivel de gestión universitaria le corresponde un adecuado nivel de práctica reflexiva; a un inadecuado nivel de investigación le corresponde un inadecuado nivel de gestión universitaria. Es necesario que el docente como profesional que es, debe tener conocimiento sobre la gestión institucional de su universidad y así servir de apoyo que está dentro de funciones; con respecto a la información sustentada en el marco teórico esta fue fundamentada por la Ley No 30220 Ley Universitaria del Capítulo VIII, en donde la gestión universitaria es una función que desarrolla el profesor universitario teniendo en cuenta la referencia a su gestión. El personal docente en la cual también se le considera investigador, como un miembro más de la institución, puede participar activamente en la gestión de su institución.

\section{CONCLUSIÓN}

Se demostró que existe relación directa entre las funciones del docente universitario y la práctica reflexiva en la facultad de psicología de la Universidad Nacional "San Luis Gonzaga" de Ica; representado en un nivel de significancia de ,000. Esto refleja que a un nivel eficiente de cumplimiento de las funciones docente le corresponde un nivel eficiente de práctica reflexiva en los docentes de la mencionada casa de estudios. 
También, se demostró que existe relación directa entre las funciones del docente universitario y el elemento cognitivo en la facultad de psicología de la Universidad Nacional "San Luis Gonzaga" de Ica; representado en un nivel de significancia de ,000. Esto refleja que a un nivel eficiente de cumplimiento de las funciones docente le corresponde un nivel eficiente del elemento cognitivo en los docentes de la mencionada casa de estudios.

Así mismo, se demostró que existe relación directa entre las funciones del docente universitario y el elemento crítico en la facultad de psicología de la Universidad Nacional "San Luis Gonzaga" de Ica; representado en un nivel de significancia de ,000. Esto refleja que a un nivel eficiente de cumplimiento de las funciones docente le corresponde un nivel eficiente del elemento crítico en los docentes de la mencionada casa de estudios.

En tal virtud, se demostró que existe relación directa entre las funciones del docente universitario y el elemento narrativo en la facultad de psicología de la Universidad Nacional "San Luis Gonzaga" de Ica; representado en un nivel de significancia de ,000. Esto refleja que a un nivel eficiente de cumplimiento de las funciones docente le corresponde un nivel eficiente del elemento cognitivo en los docentes de la mencionada casa de estudios.

Agregado a lo anterior, se demostró que existe relación directa entre la investigación y la práctica reflexiva en la facultad de psicología de la Universidad Nacional "San Luis Gonzaga" de Ica; representado en un nivel de significancia de ,109. Esto refleja que a un nivel eficiente de cumplimiento de las funciones docente le corresponde un nivel eficiente del elemento narrativo en los docentes de la mencionada casa de estudios.

Además de lo mencionado, se demostró que existe relación directa entre el mejoramiento continuo de la enseñanza y la práctica reflexiva en la facultad de psicología de la Universidad Nacional "San Luis Gonzaga" de Ica; representado en un nivel de significancia de ,000. Esto refleja que a un nivel eficiente de mejoramiento continuo de la enseñanza le corresponde un nivel eficiente de la práctica reflexiva en los docentes de la mencionada casa de estudios.

Del mismo modo, se demostró que existe relación directa entre la proyección social y la práctica reflexiva en la facultad de psicología de la Universidad Nacional "San Luis Gonzaga" de Ica; representado en un nivel de significancia de ,005. Esto refleja que a un nivel eficiente de proyección social le corresponde un nivel eficiente de la práctica reflexiva en los docentes de la mencionada casa de estudios.

Finalmente, se demostró que existe relación directa entre la gestión universitaria y la práctica reflexiva en la facultad de psicología de la Universidad Nacional "San Luis Gonzaga" de 
Ica; representado en un nivel de significancia de, 000 . Esto refleja que a un nivel eficiente de gestión universitaria le corresponde un nivel eficiente de la práctica reflexiva en los docentes de la mencionada casa de estudios.

\section{LISTA DE REFERENCIAS}

Bendezú, R. (2005). Población y Familia Rural. Huamanga Ayacucho.

Brubacher, J., Case, C., \& Reagan, T. (2000) Cómo ser un docente reflexivo. La construcción de una cultura de indagación en las escuelas. Barcelona: Gedisa.

Cerecero, I. (2018). Propuesta de un nuevo modelo: Práctica Reflexiva Mediada. Innoeduca. International Journal of Technology and Educational Innovation, 4(1), 44-53. doi: http://dx.doi.org/10.24310/innoeduca.2018.v4i1.3595

Coppola, N. (2012). Las políticas de evaluación de la función docente universitaria en el marco de la evaluación institucional: Un estudio comparado entre Argentina, España y México. (Tesis doctoral). Universidad Complutense de Madrid, España. https://eprints.ucm.es/16682/1/T34004.pdf

Domingo, A. (2013). Práctica reflexiva para docentes. De la reflexión ocasional a la reflexión metodológica. Saarbrücken (Alemania): Publicia.

García, V. (2001). La función docente del profesor universitario, su formación y desarrollo profesional. Madrid: La Muralla.

Hernández, R., Fernández, C., \& Baptista, M. (2014). Metodología de la investigación científica. (6ta Ed). Editorial McGraw-Hill. https://www.esup.edu.pe/wpcontent/uploads/2020/12/2.\%20Hernandez,\%20Fernandez\%20y\%20BaptistaMetodolog\%C3\%ADa\%20Investigacion\%20Cientifica\%206ta\%20ed.pdf

LEY No 30220 (2017). Ley universitaria. Diario Oficial El Peruano. Lima.

Martell, F. (2010). La competencia comunicativa, elemento central de la docencia. Revista Caminos abiertos, 180. http://caminosabiertos2010.blogspot.com/2010/01/lacompetencia-comunicativa-elemento.html

Oyague, M. (2014). Las funciones de docencia - investigación y extensión en la universidad nacional "Pedro Ruiz Gallo" - Lambayeque. Revista de Investigación de la Universidad Nacional de San Marcos. http://revistasinvestigacion.unmsm.edu.pe/index.php/educa/article/view/7470 
Reynaldo, V., La Torre, A., \& Frisancho, S. (2011). Percepción de la reflexión docente en un grupo de maestros de una escuela pública de Ayacucho. Educación, 20(38), 2544.

Rodriguez, R. (2013). El desarrollo de la práctica reflexiva sobre el quehacer docente, apoyada en el uso de un portafolio digital, en el marco de un programa de formación para académicos de la Universidad Centroamericana de Nicaragua. (Tesis doctoral). Universidad de Barcelona, España. http://www.redfforma.cl/documentos_sitio/43828_RRODRIGUES_TESIS.pdf

Saiz, A. y Susinos, T. (2017). Problemas pedagógicos para un Prácticum reflexivo de Maestros. Rev. complut. educ, 28(4), 993-1008. doi: https://doi.org/10.5209/RCED.50924

Santivañez, M. (2014). Sistematización de estrategias para la formación del docente reflexivo en una universidad privada de Lima. (Tesis de maestría.) Pontificia Universidad Católica del Perú, Lima. http://tesis.pucp.edu.pe/repositorio/bitstream/handle/20.500.12404/5780/SANTIVA NEZ_ARIAS_MARTHA_SISTEMATIZACION_DOCENTE.pdf?sequence $=1 \&$ is Allowed $=\mathrm{y}$

Schön, D. (1992). La formación de profesionales reflexivos. Hacia un nuevo diseño de la enseñanza y el aprendizaje en las profesiones. Barcelona: Paidós/MEC.

Shulman, L. (1987). Knowledge and teaching: Foundations of the new reform. Harvard Educational Review, 57(1), pp 1-22.

Sousa, V; Driessnack, M y Costa, I (2007). Revisión de diseños de investigación resaltantes para enfermería. Parte 1: diseños de investigación cuantitativa. Rev Latino-am Enfermagem 15(3)

Sparks-Langer, G. Y Colton, A. (1991). Synthesis of research on teachers' reflective thinking. Educational Leadership, 48 (6), 37-44.

Tolentino. (2014). Desempeño didáctico y académico del docente relacionado a la satisfacción de los estudiantes del programa de complementación pedagógica de la Universidad Nacional Mayor De San Marcos., 2013 - II. (Tesis de maestría). Universidad Nacional Mayor de San Marcos, Lima. http://cybertesis.unmsm.edu.pe/bitstream/handle/cybertesis/3981/Tolentino_sl.pdf? sequence $=1 \&$ is Allowed $=\mathrm{y}$ 
Torelló, Ó. (2011). El profesor universitario: sus competencias y formación. Profesorado. Revista de currículum y formación del profesorado. 15(3). https://www.ugr.es/ recfpro/rev153COL1.pdf

Vélez, D. y Soto, M. (2018). La función docente y la planificación para el logro del perfil de egreso en ingeniería química. Rev. cient. estud. investing, 7(1), 24-37. doi: 10.26885/rcei.7.1.24

Vergara, C. (2015). El maestro universitario y su función docente: ¿De qué función se trata? Revista «Poiésis». FUNLAM, 29, 4-5. doi: https://doi.org/10.21501/16920945.1545

Wallace, M. (2002). Training foreign language teachers: A reflective approach. USA: Cambridge University Press.

Zeichner, K. (2012). El maestro como profesional reflexivo. Conferencia presentada en el $11^{\circ}$ University of Wisconsin Reading Symposium: "Factors Related to Reading Performance", Milwaukee (Wisconsin, Estados Unidos). http://web.usbmed.edu.co/usbmed/CURSO_DOCENTE/PORTAFOLIO9/G9ARM ENIA_ARACELLY_GUZMAN_RAE.pdf 